\title{
Tanshinone I inhibits tumor angiogenesis by reducing STAT3 phosphorylation at TYR705 and hypoxia-induced HIF-1a accumulation in both endothelial and tumor cells
}

\author{
Yan Wang ${ }^{1,2}$, Jia-Xin Li ${ }^{1}$, Ying-Qing Wang ${ }^{1}$, Ze-Hong Miao ${ }^{1}$ \\ ${ }^{1}$ Division of Antitumor Pharmacology, State Key Laboratory of Drug Research, Shanghai Institute of Materia Medica, Chinese \\ Academy of Sciences, Shanghai 201203, China \\ ${ }^{2}$ College of Pharmacy, Nanchang University, Nanchang 330006, China \\ Correspondence to: \\ Ze-Hong Miao, e-mail: zhmiao@simm.ac.cn \\ Ying-Qing Wang, e-mail: yqwang@simm.ac.cn \\ Keywords: tanshinone l, angiogenesis, Stat3, HIF- $1 \alpha$, VEGF \\ Received: February 15, $2015 \quad$ Accepted: March 23, $2015 \quad$ Published: April 10, 2015
}

\section{ABSTRACT}

Tanshinone I (Tanshinone-1), a major active principle of Salvia miltiorrhiza (Danshen), has been shown to overcome tumor drug resistance and metastasis. Here we report that tanshinone-1 inhibits angiogenesis. Tanshinone-1 inhibited proliferation, migration and tube formation of vascular endothelial cells, rat aortic ring sprouting and the neovascularization of the chick chorioallantoic membrane in a concentration-dependent manner. In endothelial cells, tanshinone-1 almost completely inhibited phosphorylation of Stat3 at Tyr705 regardless of hypoxia or normoxia but only slightly decreased the hypoxia-induced HIF-1a accumulation. In tumor cells, contrastively, tanshinone-1 could not only make phosphorylation of Stat3 at Tyr705 disappear but also reduce the hypoxia-induced accumulation of HIF-1a to its baseline levels at normoxia. Consequently, VEGF secretion from tumor cells was reduced, which could potentiate the direct inhibition of tanshinone-1 on endothelial cells. Together with its overcoming tumor drug resistance and metastasis, our results reveal unique characteristics of tanshinone-1 and its improved derivatives as promising angiogenesis inhibitors.

\section{INTRODUCTION}

Angiogenesis inhibition has become an important strategy for cancer therapy. More than 10 angiogenesis inhibitors have been used in the clinic. Most of them, either antibody agents or small-molecule drugs, target the vascular endothelial growth factor (VEGF) - VEGF receptor (VEGFR) signaling pathway. However, several issues have arisen from their applications. They offer only very limited incremental survival benefits, elicit severe toxicities especially cardiovascular toxicities and possibly stimulate treatment resistance and metastasis [1]. These issues drive explorations on targeting other drivers of angiogenesis, for instance, angiopoietin. Till now, unfortunately, the efforts have been rewarded with only limited successes. In Phase III trials in patients with ovarian cancer, the angiopoietin inhibitor trebananib increased the median overall survival by only 1 month
$[2,3]$. Therefore, finding new antiangiogenesis strategies becomes urgent for cancer therapy in a safe, efficacious way and ideally overcoming tumor drug resistance and/ or metastasis.

Many natural products have been reported to inhibit angiogenesis via various mechanisms though having scarcely been approved for cancer therapy yet due to toxicities or other reasons [4-10]. The traditional Chinese medicine Salvia miltiorrhiza (Danshen) is famous for its safe, effective treatment of cardiovascular diseases with a long history. Its several preparations are still widely used, especially in the treatment of angina pectoris and congestive heart failure in China [11-14]. Tanshinone I (Tanshinone-1; Figure 1A), an active principle of Danshen, shows its clinical safety based on its high content in this plant [11] and its cardiovascular activity [12]. More importantly, tanshinone-1 has been shown to kill drugresistant tumor cells. This activity is correlated well 
B

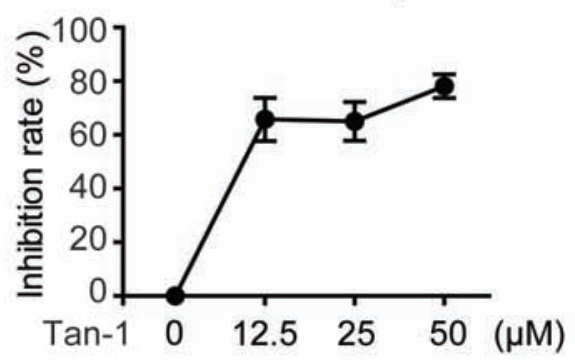

A<smiles>Cc1coc2c1C(=O)C(=O)c1c-2ccc2c(C)cccc12</smiles>

C

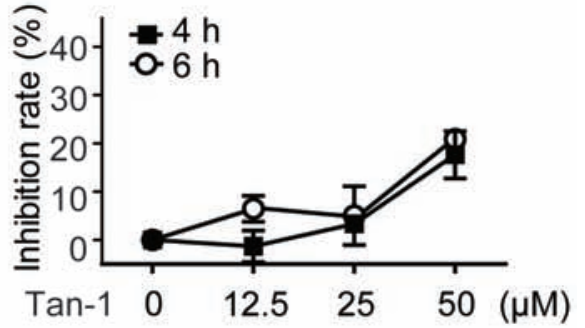

D
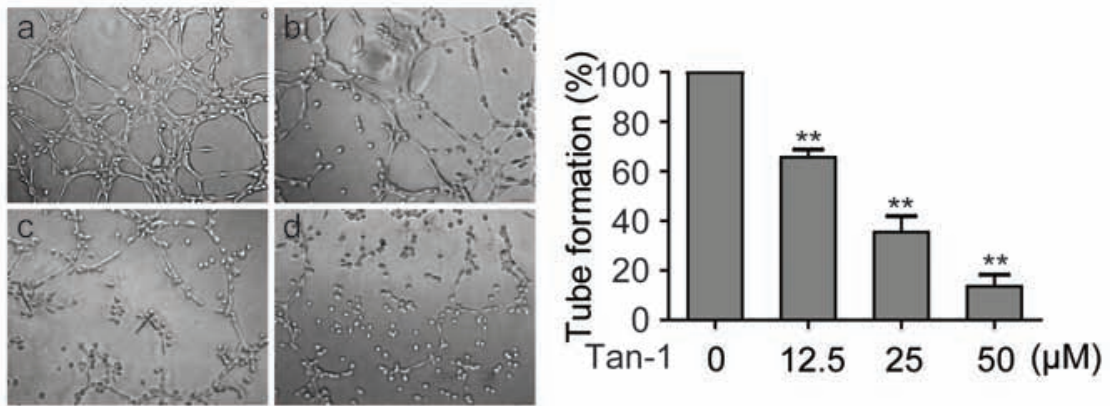

E
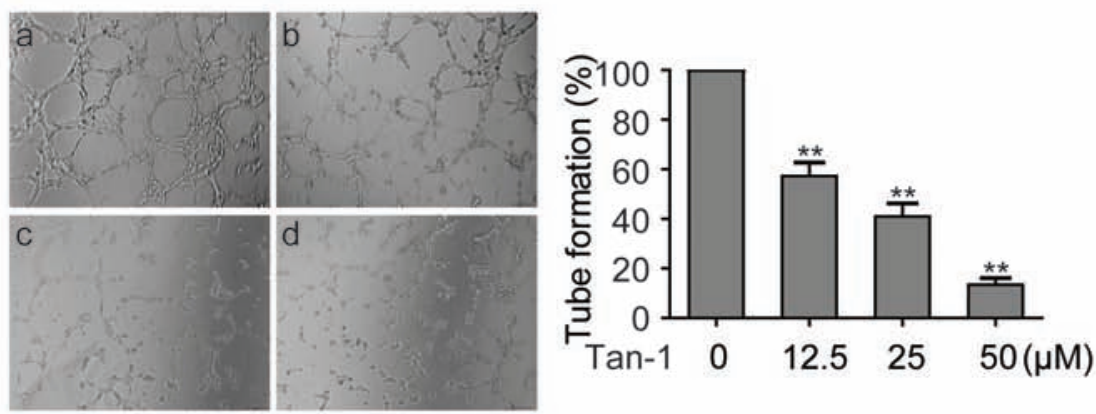

F
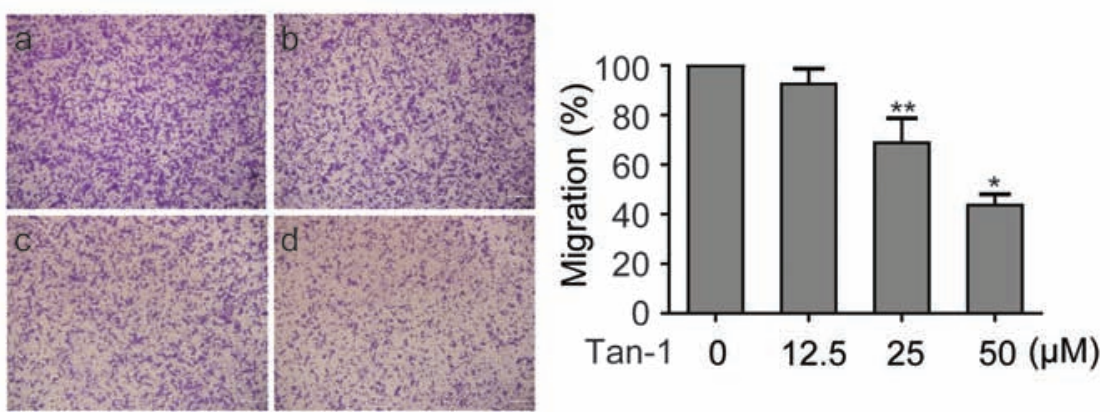

Figure 1: Tanshinone-1 (Tan-1) inhibits the tube formation and migration of endothelial cells. A. Chemical structure of Tan-1. B. Tan-1 inhibited proliferation of endothelial cells. Human microvascular endothelial HMEC-1 cells were exposed to gradient concentrations of Tan-1 for $72 \mathrm{~h}$. Proliferation inhibition of the cells was measured by the CellTiter-Glo ${ }^{\circledR}$ Luminescent Cell Viability assay. C. Tan-1 inhibited proliferation of endothelial cells at the same cell density and exposure time to $\mathbf{D}, \mathbf{E}$ or $\mathbf{F}$. $\mathbf{D}$ to $\mathbf{F}$. Tan-1 inhibited the tube formation of both HMEC-1 cells (D) and HUVEC cells $(\mathbf{E})$ and the migration of HMEC-1 cells $(\mathbf{F})$. Cells were treated with vehicle (a), or Tan-1 $12.5 \mu \mathrm{M}$ (b), $25 \mu \mathrm{M}$ (c) or $50 \mu \mathrm{M}$ (d) in the corresponding left panel for $4 \mathrm{~h}$ (D and $\mathbf{E})$ or $6 \mathrm{~h}(\mathbf{F})$. Magnification: $20 \times(\mathbf{D})$ or $10 \times(\mathbf{E}$ and $\mathbf{F})$. The data from three independent experiments were expressed as mean $\pm \mathrm{SD}$ in the corresponding right panel. $* p<0.05 ; * * p<0.01$. 
with its reducing the active form of signal transducer and activator of transcription 3 (Stat3), phosphorylated Stat3 at Tyr705 (p-705-Stat3) [11]. Tanshinone-1 was also found to inhibit tumor metastasis by suppressing the tumor necrosis factor- $\alpha$ (TNF- $\alpha)$-induced transcriptional activity of nuclear factor kappa B (NFאB) [15].

Here we show that tanshinone-1 inhibits angiogenesis at either hypoxia or normoxia by directly acting on both endothelial and tumor cells. Tanshinone-1 inhibited proliferation, migration and differentiation (tube formation) of endothelial cells and thus blocked angiogenesis at its initiation stage. The antiangiogenic activity was further reflected in its suppressing rat aortic ring sprouting and the neovascularization of the chick chorioallantoic membrane. The effect of tanshinone-1 on endothelial cells was correlated mainly with its reducing p-705-Stat 3 at both hypoxia and normoxia though it also slightly lowered the hypoxia-induced accumulation of hypoxia inducible factor 1 alpha (HIF-1 $\alpha)$. Moreover, this effect could be further amplified by the reduction of VEGF secretion from tumor cells subsequent to tanshinone1-mediated decrease in p-705-Stat3 regardless of ambient oxygen conditions and hypoxia-induced HIF-1 $\alpha$ accumulation. Together with its good safety and excellent characteristics in overcoming tumor drug resistance and metastasis, our findings could distinguish tanshinone-1 and its improved derivatives from present antiangiogenesis agents, especially those used in the clinic.

\section{RESULTS}

\section{Tanshinone-1 inhibits proliferation, tube formation and migration of vascular endothelial cells}

Vascular endothelial cells play critical roles in angiogenesis, especially at its initiation stage. Tanshinone-1 was shown to inhibit proliferation of human microvascular endothelial (HMEC-1) cells in a concentration-dependent manner (Figure 1B). For the 72-h treatment, tanshinone- 1 had an $\mathrm{IC}_{50}$ value of $7.75 \mu \mathrm{M}$ in HMEC-1 cells, which is roughly equal to its previously reported potency in tumor cells [11]. To find the proper conditions to test its effect on the tube formation and migration of vascular endothelial cells, we exposed HMEC- 1 cells $\left(2.5 \times 10^{4}\right.$ cells or $2 \times 10^{5}$ cells per well $)$ to tanshinone- 1 for $4 \mathrm{~h}$ or $6 \mathrm{~h}$. Tanshinone- 1 displayed only marginal proliferation inhibition on HMEC-1 cells, and even at $50 \mu \mathrm{M}$, the inhibitory rate was just below $20 \%$ (Figure 1C). At the same cell density and exposure time, however, tanshinone-1 caused suppression of the tube formation of both HMEC-1 cells (Figure 1D) and human umbilical vascular endothelial (HUVEC) cells (Figure 1E) and the migration of HMEC-1 cells (Figure 1F) in a concentration-dependent manner. Proliferation could provide enough cell number of endothelial cells; migration could allow those cells to move themselves to new locations; and differentiation (here mimicked by the tube formation assay) is required for single endothelial cells to evolve finally into vascular networks. All of them are important to initiate angiogenesis. Therefore, our results suggest that tanshinone-1 could inhibit the initiation of angiogenesis at its different essentials.

\section{Tanshinone-1 inhibits angiogenesis both ex vivo and in vivo}

Assays for rat aortic ring sprouting and neovascularization of the chick chorioallantoic membrane are classical models for ex vivo and in vivo angiogenesis, respectively. Tanshinone-1 was shown to reduce both the microvessel sprouting of rat aortic rings (Figure 2A) and the microvessels of the chick chorioallantoic membrane (Figure 2B, the right panel vs the left one of each image) in a concentration-dependent manner. The results further indicate that tanshinone-1 could suppress not only the initiation of angiogenesis by inhibiting the functions of endothelial cells but also the final formation of blood vessels.

\section{Tanshinone-1 reduces p-705-Stat 3 and HIF-1 $\alpha$ in endothelial cells, which could contribute to its tube-formation inhibition}

Both Stat3 and HIF-1 $\alpha$ have been reported to be involved in angiogenesis [16, 17]. Tanshinone-1 was shown to reduce the levels of p-705-Stat3 at both normoxic and hypoxic conditions, but not to lower the levels of either the total Stat3 or phosphorylated Stat3 at Ser727 (p-727-Stat3) in HMEC-1 cells (Figure 2C). In contrast, tanshinone-1 could also decrease the levels of hypoxia-induced HIF-1 $\alpha$ protein in the same cells in a concentration-dependent manner, but apparently weakly when compared with its effect on p-705-Stat3 (Figure 2D).

To detect whether the antiangiogenic activity of tanshinone- 1 is linked to its effect on p-705-Stat3 or HIF-1 $\alpha$ protein, we used specific siRNA against hif-1 $\alpha$ (siHIF$1 \alpha-1$ and -2 with different sequences), stat 3 (siStat3-1 and -2 with different sequences), or both to silence the corresponding genes (Figure 3A). Tanshinone-1 was shown to inhibit the tube formation of HMEC-1 cells with nearly equal potency at both normoxia and hypoxia (Figure 3B and 3C; Supplementary Figure S1A and S1B). Silencing of the hif-1 $\alpha$ gene did not obviously impair the tube formation of HMEC-1 cells at either normoxia or hypoxia, but slightly potentiated the inhibitory effect of tanshinone-1 (Figure 3B and 3C; Supplementary Figure $\mathrm{S} 1 \mathrm{~A}$ and $\mathrm{S} 1 \mathrm{~B})$. In contrast, silencing of the stat 3 gene or both stat 3 and hif- $1 \alpha$ genes reduced the tube formation at both normoxia and hypoxia, which was further potentiated 
A
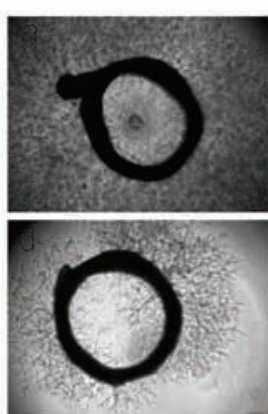

B
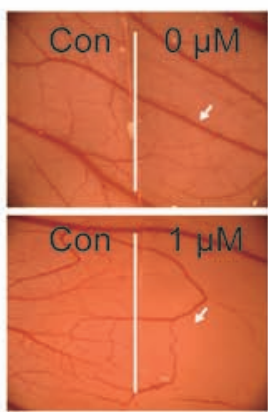
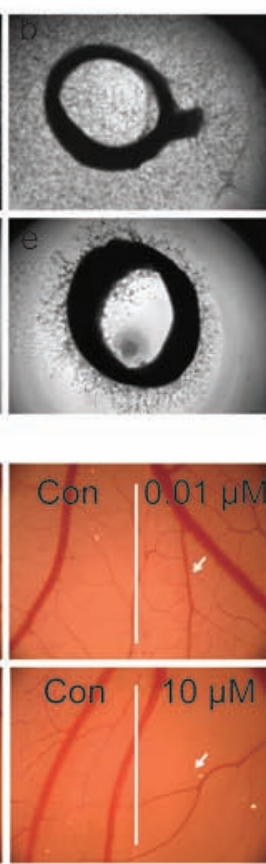
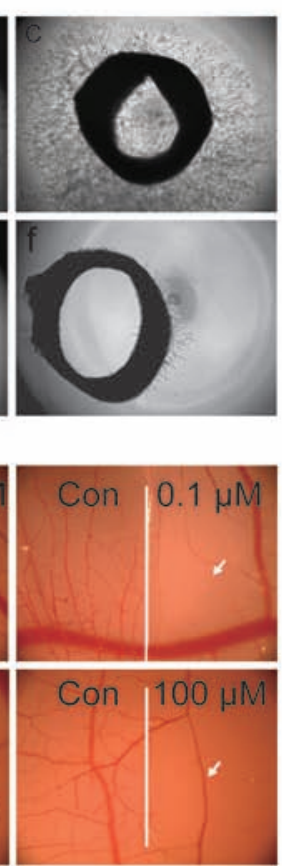

C

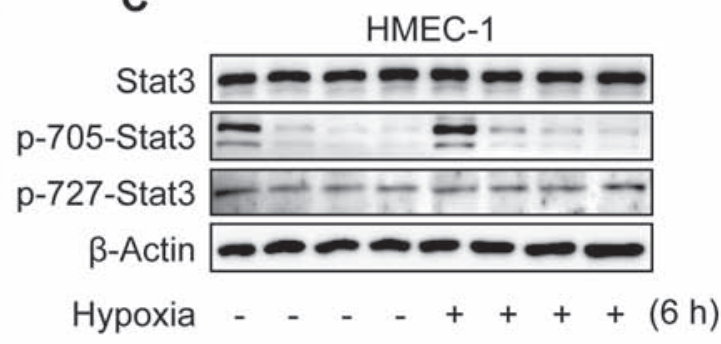

Tan-1 $012.52550012 .52550(\mu \mathrm{M})$

D

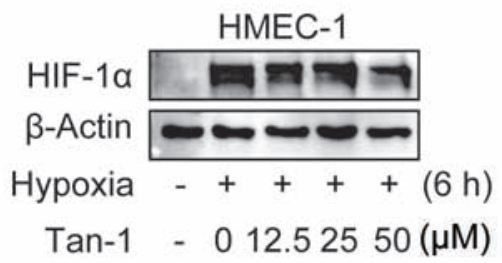

Figure 2: Tanshinone-1 (Tan-1) inhibits angiogenesis and reduces p-705-Stat3 and HIF-1 $\alpha$ in HMEC-1 cells. A. Rat aortic rings were treated with vehicle (a), Tan-1 $1.25 \mu \mathrm{M}$ (b), $2.5 \mu \mathrm{M}$ (c), $5 \mu \mathrm{M}$ (d), $10 \mu \mathrm{M}$ (e), or $20 \mu \mathrm{M}$ (f) for 7 days; magnification: $4 \times$. B. Chicken eggs were treated with vehicle (the left hand side of each image) or gradient concentrations of Tan-1 (the right hand side of each image); magnification: 1.6×. C and D. Tan-1 reduced p-705-Stat3 C and HIF-1 $\alpha$ D in HMEC-1 cells. Cells were treated with gradient concentrations of Tan- 1 for $6 \mathrm{~h}$ at hypoxia or normoxia, and then detected by Western blotting. All experiments were independently done at least 2 times and the representative images were presented.

by the treatment with $50 \mu \mathrm{M}$ tanshinone-1 (Figure 3B and 3C; Supplementary Figure S1A and S1B). These results demonstrate the following facts: (1) Ambient oxygen concentrations does not affect the inhibitory effect of tanshinone-1 on the tube formation of HMEC-1 cells, (2) Stat3 rather than HIF-1 $\alpha$ stimulates the tube formation of HMEC-1 cells at both normoxia and hypoxia, and (3) Downregulation of Stat3 or HIF-1 $\alpha$ or both could not reverse but slightly potentiates the inhibitory effect of tanshinone-1 on tube formation of endothelial cells.

\section{Tanshinone-1 decreases the levels of HIF-1a and p-705-Stat 3 and the secretion of VEGF in tumor cells}

VEGF, the most potent angiogenesis-stimulating factor, can be secreted from solid tumor cells in response to hypoxia stress and/or active angiogenic growth factor signaling such as the Stat3 signaling. Reduction of VEGF secretion from tumor cells might attenuate angiogenesis [18]. When MCF-7 cells were exposed to hypoxia, the level of HIF-1 $\alpha$ first rose, peaked at $6 \mathrm{~h}-9 \mathrm{~h}$, and then declined gradually (Figure 4A). Tanshinone-1 was revealed to lower the 6-h-hypoxia-induced accumulation of HIF-1 $\alpha$ protein in a concentration-dependent manner in different tumor cells including MCF-7, SKOV3, HCC1937, A549 and HeLa cells (Figure 4B and 4C). And its activity in reducing HIF- $1 \alpha$ in tumor cells was more potent than in endothelial cells (Figure 2D). In contrast, tanshinone-1 elicited similar effects on Stat 3 in both MCF-7 and HMEC-1 cells by decreasing p-705-Stat3 but not lowering the levels of total Stat3 or p-727-Stat3 at normoxia or hypoxia (Figure 4D). Tanshinone-1 was further shown to reduce the hypoxia-induced increase in the levels of VEGF mRNA and VEGF protein secretion in MCF-7 cells (Figure 4E and 4F).

\section{Tanshinone-1 decreases hypoxia-induced HIF-1 $\alpha$ protein through the proteasome pathway in MCF-7 cells}

Hypoxia induces the accumulation of HIF- $1 \alpha$ protein mainly by blocking its degradation via the ubiquitin proteasome pathway [19]. Treatments with tanshinone-1 did not change the mRNA level of hif- $1 \alpha$ (Figure 5A) but decreased the protein levels of HIF-1 $\alpha$ in MCF-7 cells (Figure 5B). MG-132, a widely used proteasome inhibitor, could enhance the levels of HIF-1 $\alpha$ protein, especially prominently at normoxia (Figure 5B), just as previously reported [19]. Pretreatments with 
A
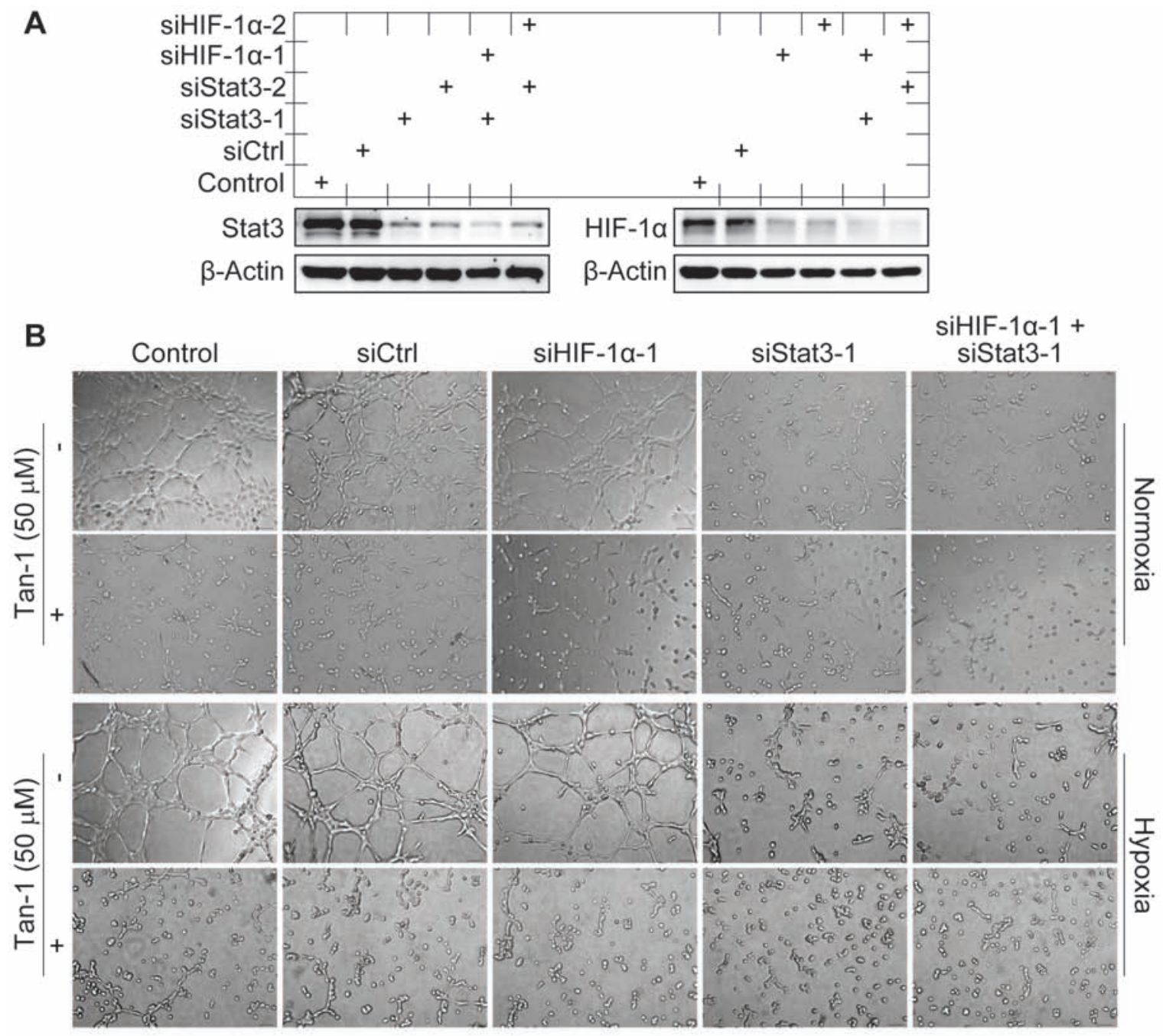

C

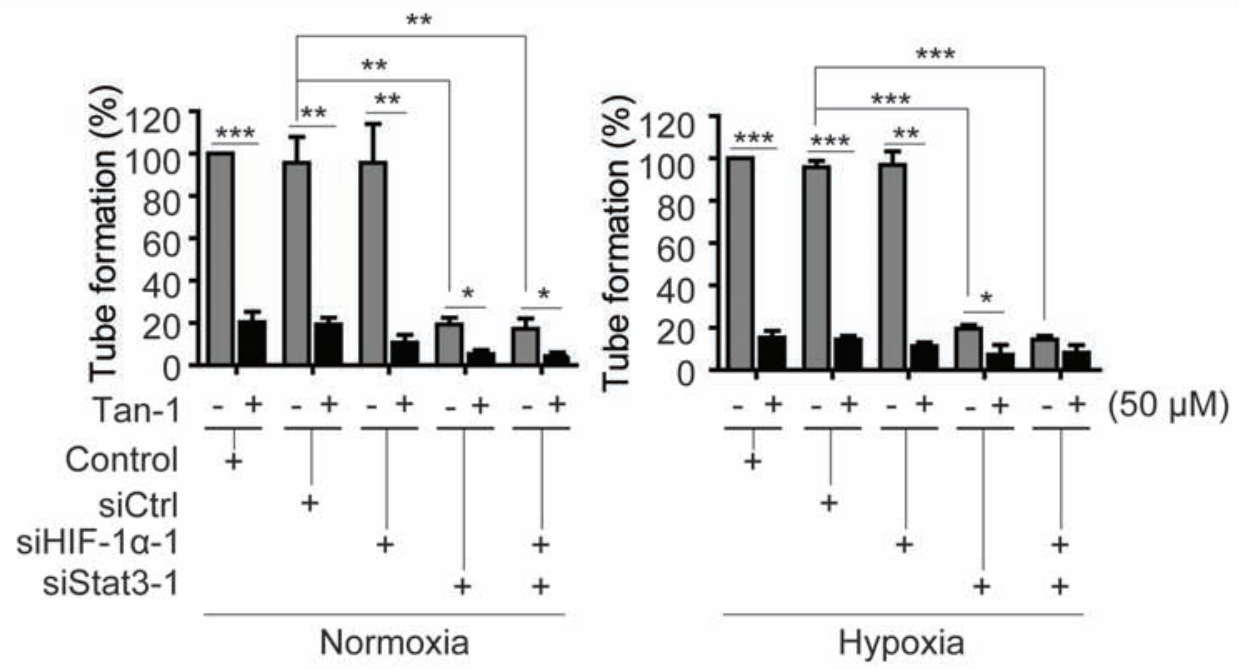

Figure 3: Effects of Stat3 and/or HIF-1 $\alpha$ downregulation on the tube-formation inhibition of tanshinone-1 (Tan-1) in HMEC-1 cells. A. HMEC-1 cells were treated with scramble siRNA (siCtrl), specific siRNA targeting human hif-1 $\alpha$ cDNA (siHIF$1 \alpha-1$ and -2 ) or stat 3 cDNA (siStat3-1 and -2) or just the transfection reagent (Control) for $48 \mathrm{~h}$. And then the cells were detected by Western blotting for the levels of Stat 3 and HIF-1 $\alpha$ proteins. B. Following the 48-h-pretreatment of HMEC-1 cells with siCtrl, siHIF-1 $\alpha-1$, siStat3-1 or the transfection reagent (Control), the cells treated with or without $50 \mu \mathrm{M}$ Tan-1 were seeded on Martigel and allowed to form tube networks for $4 \mathrm{~h}$ at normoxia or hypoxia; magnification: $20 \times$. C. Data from three independent experiments in B were expressed as mean \pm SD. ${ }^{*} p<0.05 ; * * p<0.01$; and ${ }^{* * *} p<0.001$. 
A

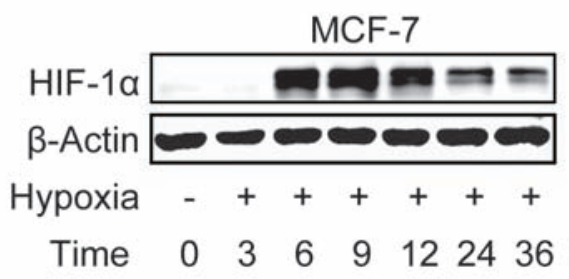

B

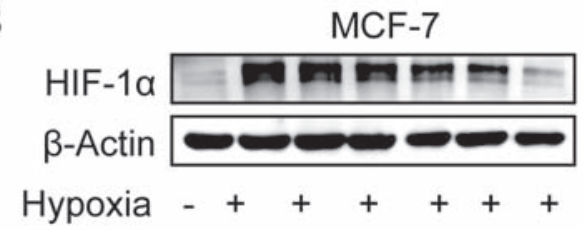

Tan-1 $003.1256 .2512 .52550(\mu \mathrm{M})$

C

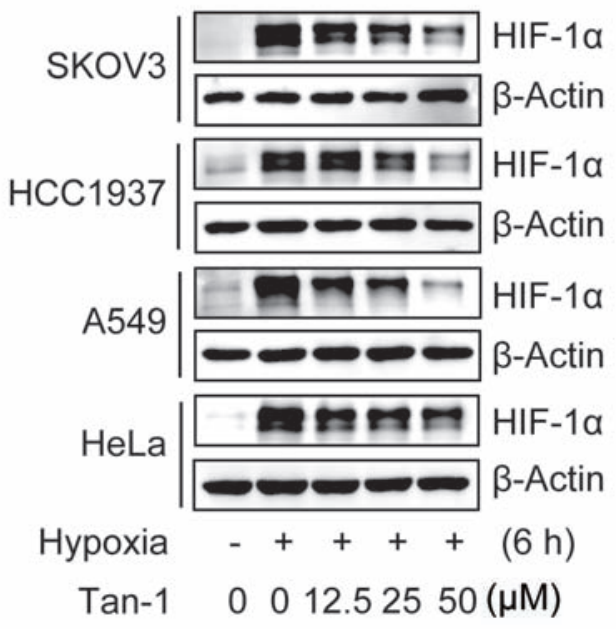

D

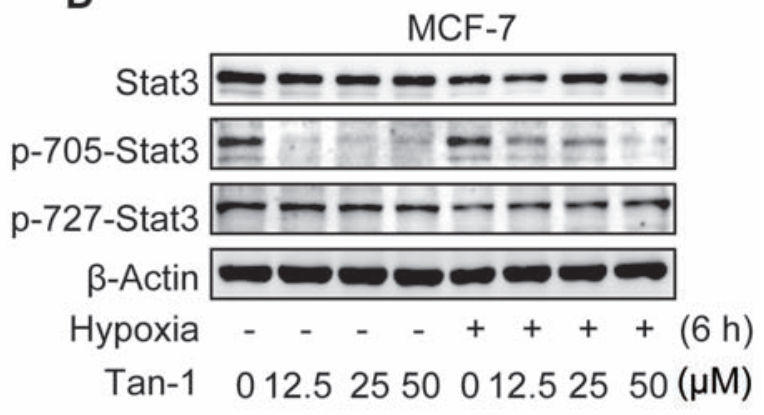

E

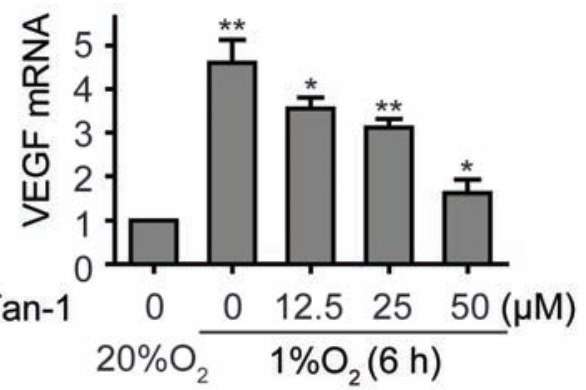

$\mathbf{F}$

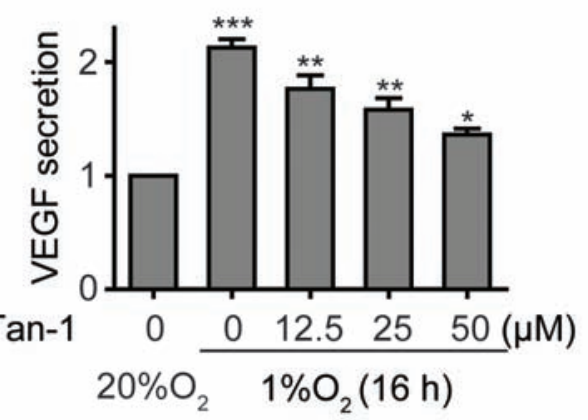

Figure 4: Tanshinone-1 (Tan-1) reduces the levels of HIF-1 $\alpha$ and p-705-Stat3 and decreases the mRNA level and protein secretion of VEGF in hypoxic tumor cells. A. MCF-7 cells were exposed to hypoxia $\left(1 \% \mathrm{O}_{2}\right)$ for the indicated time, and HIF-1 $\alpha$ levels were analyzed by Western blotting. B and C. MCF-7 B, SKOV3, HCC1937, A549 and HeLa C cells were treated with gradient concentrations of Tan-1, and immediately exposed to hypoxia for 6 h. HIF-1 $\alpha$ levels were detected by Western blotting. D. Tan-1 reduced p-705-Stat3 in MCF-7 cells at either normoxia or hypoxia. The cells were treated with gradient concentrations of Tan-1, and immediately exposed to hypoxia or normoxia for $6 \mathrm{~h}$. Western blotting was done to detect the levels of Stat3 and p-705-Stat3 or p-727-Stat3. E and F. Tan-1 inhibited vegf gene transcription (E) and VEGF protein secretion (F) in hypoxic MCF-7 cells. The cells were treated with gradient concentrations of Tan-1 at hypoxia for $6 \mathrm{~h}$ (vegf transcription) or $16 \mathrm{~h}$ (VEGF secretion). Then the total RNA was isolated for vegf mRNA analyses by Real-Time PCR, and cell supernatants were collected and measured for VEGF protein secretion by ELISA. The level of mRNA or protein at normoxia $\left(20 \% \mathrm{O}_{2}\right)$ was normalized as 1 . Data from three independent experiments were expressed as mean $\pm \mathrm{SD} .{ }^{*} p<0.05 ;{ }^{* *} p<0.01 ;{ }^{* *} p<0.001$.

MG-132 effectively reversed the tanshinone-1-driven decrease of HIF-1 $\alpha$ protein (Figure 5B). The data indicate that tanshinone-1 could decrease hypoxia-induced HIF-1 $\alpha$ protein by impairing the proteasome-mediated degradation rather than by reducing the expression of the hif- $1 \alpha$ gene.

\section{Both HIF-1 $\alpha$ and Stat 3 signaling contributes to the tanshinone-1-driven reduction of the mRNA levels and the protein secretion of VEGF in hypoxic MCF-7 cells}

To examine whether the effect of tanshinone- 1 on VEGF is correlated with its effect on p-705-Stat3 and/or
HIF-1 $\alpha$ protein in tumor cells, we used specific siRNA against hif-1 $\alpha$ (siHIF- $1 \alpha-1$ and -2), stat3 (siStat3-1 and -2) or both to downregulate the expression of the corresponding genes in MCF-7 cells (Figure 5C). We found that hypoxia induced the increase in the mRNA levels and the protein secretion of VEGF, which could be obviously inhibited by tanshinone-1 (Figure 4E and 4F; the groups of Control and siCtrl in Figure 5D and 5E and in Supplementary Figure S2A and S2B). Downregulation of either HIF-1 $\alpha$ or Stat3 partially prevented the hypoxiainduced increase in the mRNA levels and the protein secretion of VEGF, which was further potentiated by adding tanshinone-1. In contrast, simultaneous 
A

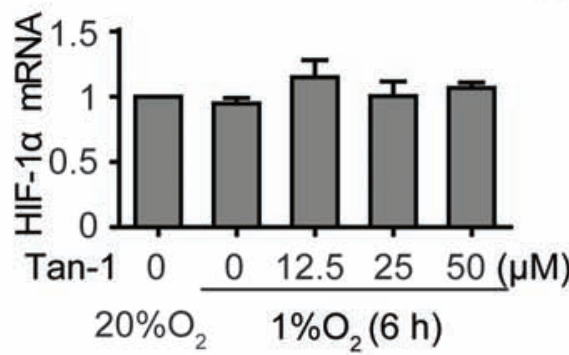

B

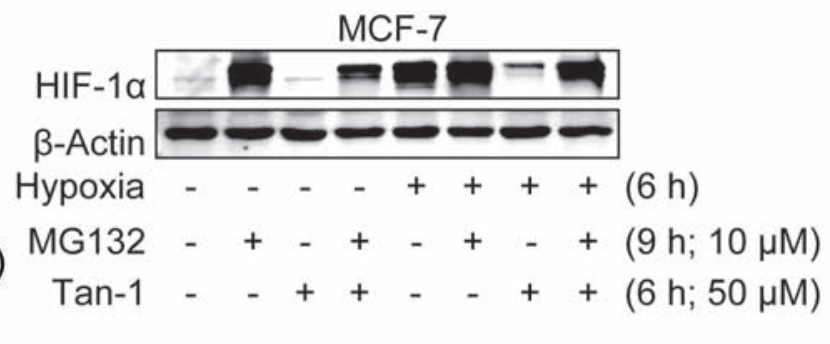

C

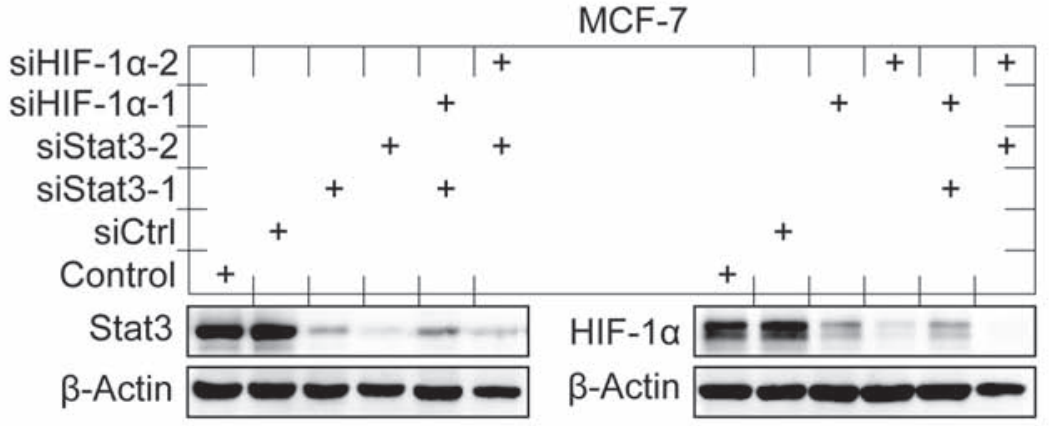

D
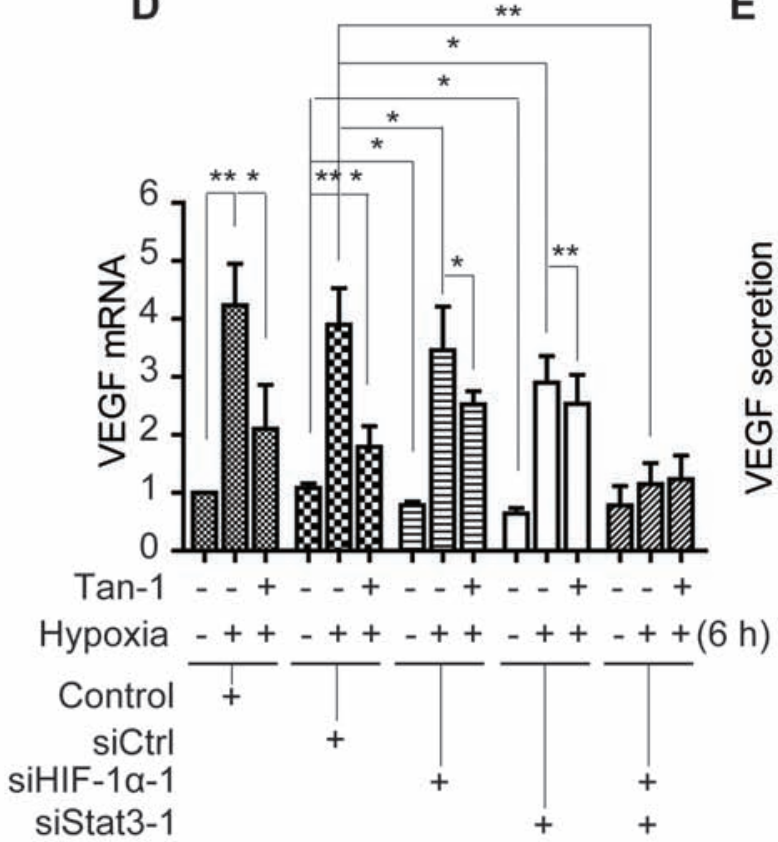

E

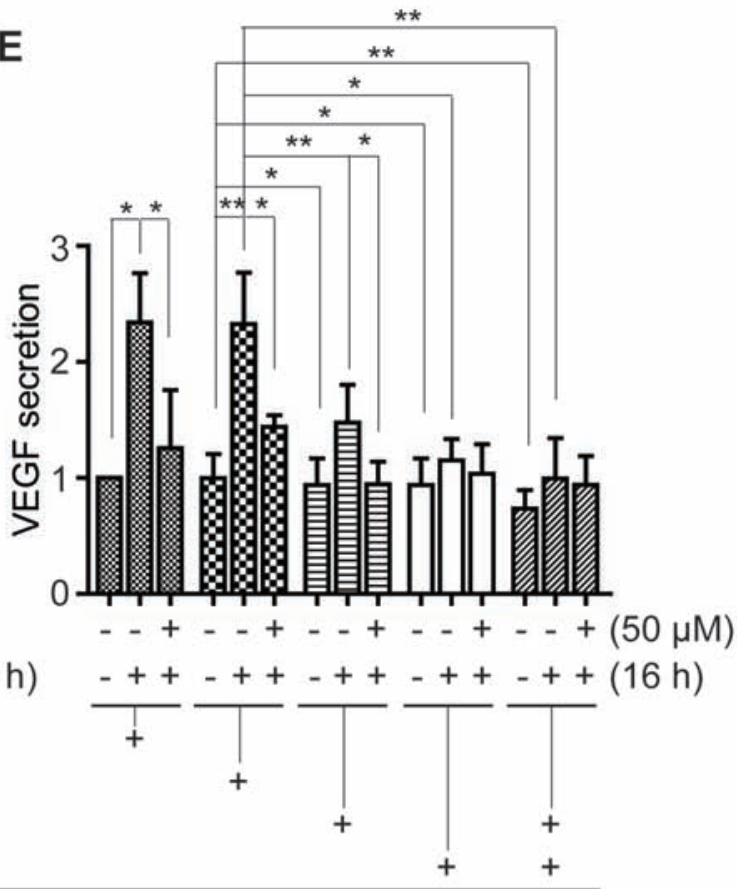

Figure 5: Downregulation of HIF-1 $\alpha$ and Stat3 alleviates the reduction of VEGF mRNA and protein secretion induced by tanshinone-1 (Tan-1) in hypoxic MCF-7 cells. A. MCF-7 cells were treated with gradient concentrations of Tan-1, and immediately exposed to hypoxia for $6 \mathrm{~h}$. The levels of hif- $1 \alpha \mathrm{mRNA}$ were analyzed by Real-Time PCR. B. Cells were pretreated with MG-132 for $3 \mathrm{~h}$, then treated with $50 \mu \mathrm{M}$ Tan-1 and exposed to hypoxia or normoxia for another $6 \mathrm{~h}$, respectively. The cells were collected for Western blotting. C. MCF-7 cells were pretreated with the transfection reagent (Control) or siRNA (siCtrl, siHIF-1 $\alpha-1$ or -2, siStat3-1 or -2 as indicated) for $48 \mathrm{~h}$. Western blotting was done to detect the levels of Stat 3 and HIF-1 $\alpha$ proteins. D and E. Followed by the 48-h pretreatment with the indicated siRNA, the cells were treated with $50 \mu \mathrm{M}$ Tan-1 and exposed to hypoxia for $6 \mathrm{~h}(\mathbf{D})$ or $16 \mathrm{~h}(\mathbf{E})$. Then Real-Time PCR and ELISA assays were done to detect the mRNA levels D and protein secretion E of VEGF, respectively. The level of mRNA or protein in the control group at normoxia was normalized as 1. Data from three independent experiments were expressed as mean \pm SD. $* p<0.05 ; * * p<0.01 ; * * * p<0.001$. 
downregulation of both HIF-1 $\alpha$ and Stat3 completely prevented the hypoxia-induced VEGF increase. Moreover, the addition of tanshinone-1 did not further lower the levels of the VEGF mRNA or the secreted VEGF protein (Figure 5D and 5E; Supplementary Figure S2A and S2B). These results indicate that both HIF- $1 \alpha$ and Stat 3 signaling contributes to the effect of tanshinone-1 on the mRNA levels and the protein secretion of VEGF in hypoxic MCF7 cells.

\section{DISCUSSION}

In this study, we demonstrate that tanshinone-1 inhibits tumor angiogenesis in both direct and indirect ways. Tanshinone-1 directly suppresses proliferation, migration and tube formation (differentiation) of endothelial cells, which could be amplified indirectly by its reducing the secretion of VEGF from tumor cells. Tanshinone- 1 attenuates the sprouting of rat aortic rings and the neovascularization of the chick chorioallantoic membrane. The data suggest that tanshinone-1 could not only block the endothelial-cell-mediated initiation stage but also impair the whole process of angiogenesis. In solid tumors, besides, tanshinone-1 reduces VEGF paracrine from tumor cells, which might prevent the stimulation of VEGF on endothelial cells (Figure 6), further strengthening its antiangiogenesis activity.
Both Stat3 and HIF-1 $\alpha$ have been revealed to drive tumor angiogenesis, metastasis and drug resistance [20-22]. We previously reported that reduction of p-705-Stat 3 or HIF- $1 \alpha$ accumulation could overcome drug resistance $[6,11]$. Stat3 has also been shown to form transcription complexes with $\mathrm{NF} \kappa \mathrm{B}$, which synergistically drives the expression of the metastasis-driving factors [23]. So tanshinone-1-induced reduction of p-705-Stat 3 might also contribute to its reported antimetastasis activity [15]. In this study, we show that tanshinone- 1 decreases p-705-Stat3 regardless of the ambient oxygen conditions in both endothelial and tumor cells. Moreover, downregulation of Stat 3 abrogates tube formation of HMEC-1 cells and VEGF secretion from MCF-7 cells, which is further potentiated by tanshinone-1. These data suggest a possibility that tanshinone- 1 could inhibit angiogenesis through the whole solid tumor, including its hypoxic and non-hypoxic regions.

Stat 3 has been demonstrated to directly promote the expression of HIF-1 $\alpha[22,24]$. However, the tanshinone1 -driven reduction of hypoxia-induced HIF-1 $\alpha$ accumulation could not be correlated with its effect on Stat 3 in either endothelial or tumor cells. In the identicallytreated endothelial cells, tanshinone-1 reduces $\mathrm{p}-705-\mathrm{Stat} 3$ but only slightly lowers HIF-1 $\alpha$ accumulation. Abrogating the expression of HIF-1 $\alpha$ does not block the tube formation or obviously change the effect of tanshinone-1

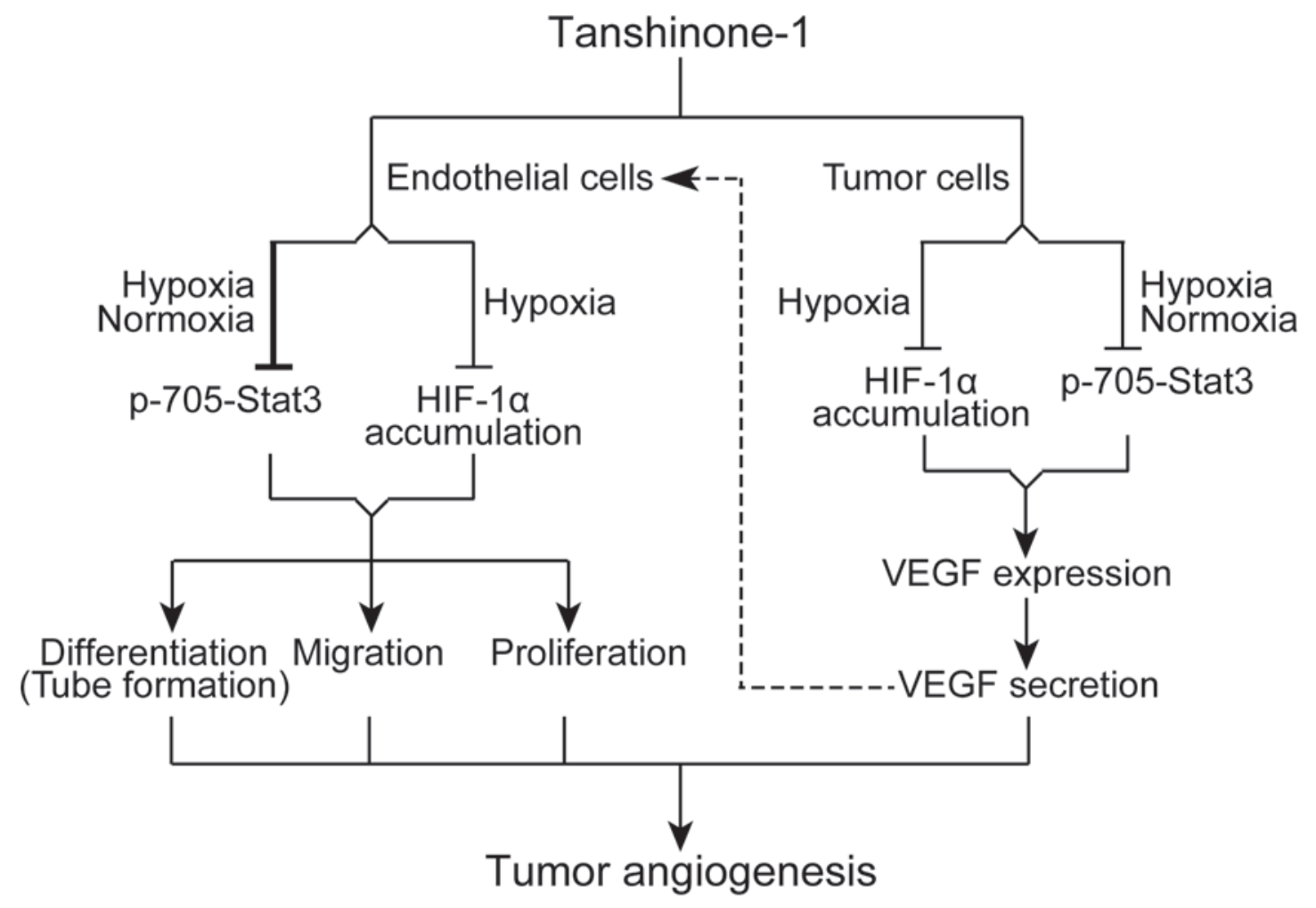

Figure 6: Schematic presentation of possible mechanisms by which tanshinone-1 inhibits tumor angiogenesis. 
on the tube formation either. Similarly in tumor cells, tanshinone-1 does not alter the mRNA levels of HIF-1 $\alpha$ in tumor cells whereas the proteosome inhibitor MG-132 prevents the reduction of HIF-1 $\alpha$ accumulation induced by tanshinone-1. These data indicate that tanshinone-1 inhibits Stat 3 and HIF-1 $\alpha$ possibly by independent mechanisms. In contrast, these independent changes in Stat 3 and HIF- $1 \alpha$ induced by tanshinone- 1 synergistically inhibit the tube formation of endothelial cells and the VEGF secretion from tumor cells.

Together, as an angiogenesis inhibitor, tanshinone-1 displays unique characteristics as follows. 1) Tanshinone-1 reduces $\mathrm{p}$-705-Stat3 possibly by certain unknown approach(es) different from those used by the known Stat3 inhibitors [25] as we reported previously [11]. 2) Tanshinone-1 lowers the accumulation of HIF-1 $\alpha$, a principal regulator of hypoxia, in tumor cells, which could alleviate the ill biological effects of hypoxia. On the other hand, its direct tumor cell killing might ease the demand for oxygen in the tumor. Both help to improve tumor treatment [1]. 3) Tanshinone-1 has been shown to overcome tumor drug resistance [11] and inhibit metastasis [15], which might help to prevent and/or delay treatment resistance and metastasis resulting from antiangiogenic therapy [1]. 4) Importantly, tanshinone-1 is one of major active ingredients of Danshen, and Danshen has been safely used in the clinic for a very long time. Moreover, tanshinone-1 itself possesses therapeutic effects on cardiovascular diseases [12]. Thus, tanshinone-1 might have good safety and especially avoid causing severe cardiovascular toxicities that frequently occur in the use of most antiangiogenic agents at present. These characteristics could be reasonable to make tanshinone-1 distinct from other angiogenesis inhibitors. However, its drug development as an angiogenesis inhibitor requires further demonstrating its molecular mechanisms and improving its biological activity and physicochemical properties, both of which are underway.

\section{MATERIALS AND METHODS}

\section{Cell culture}

Human microvascular endothelial HMEC-1 cells were obtained from the French National Institute of Health and Medical Research (INSERM, FR), and cultured with complete MCDB131 medium (Invitrogen, Carlsbad, CA, USA). HUVEC cells were purchased from AllCells (Shanghai, China). Human breast cancer MCF-7 and HCC1937, lung cancer A549, cervical cancer HeLa and ovarian cancer SKOV3 cells were all purchased from the American Type Culture Collection (ATCC, Manassas, VA, USA). Cells were normally cultured in the ATCC-specified medium in humidified atmosphere containing $5 \% \mathrm{CO}_{2}$ at $37^{\circ}$. Hypoxia treatments were performed by putting the tested cells in a humidified atmosphere containing 5\% $\mathrm{CO}_{2}$ at $1 \%$ oxygen partial pressure (Ruskin Invivo 400 system; Ruskin Technology Ltd, Bridgend, UK).

\section{Drugs}

Tanshinone-1 and MG-132 were purchased from Selleck (Houston, TX, USA). Antibodies against p-705-Stat3, p-727-Stat3 and Stat3 were purchased from Cell Signaling Technology (Beverly, MA, USA). The antibody against HIF-1 $\alpha$ and the growth factor reduced matrigel (BD354230) were obtained from BD Biosciences (Becton Dickinson Labware, MA). The antibody against $\beta$-Actin was purchased from Santa Cruz Biotechnology (Santa Cruz, CA, USA).

\section{Proliferation inhibition assays}

Proliferation-inhibitory effects of tanshinone-1 on HMEC-1 cells were assessed by the CellTiter-Glo ${ }^{\circledR}$ Luminescent Cell Viability Assay as reported previously [26]. HMEC- 1 cells $\left(8 \times 10^{3}\right.$ cells per well for a $72-\mathrm{h}$ assay) were seeded to 96-well plates for attachment overnight (for the 72-h assay). Then the cells were treated with gradient concentrations of tanshinone- 1 for $72 \mathrm{~h}$. On the other hand, to find the proper experimental conditions for the following tube formation and cell migration assays, HMEC- 1 cells $\left(2 \times 10^{5}\right.$ cells per well for a 6 -h assay and $2.5 \times 10^{4}$ cells per well for a 4 -h assay) were seeded to 96-well plates and then treated with gradient concentrations of tanshinone-1 immediately for $4 \mathrm{~h}$ or $6 \mathrm{~h}$. The cell viability was examined by using the CellTiterGlo $^{\circledR}$ Luminescent Cell Viability Assay kit (Promega, Madison, WI). Luminescence was recorded with an EnVision Multilabel Reader (PerkinElmer, Waltham, MA).

\section{Tube formation assays}

Chilled liquid Matrigel was dispensed onto 96-well plates $\left(50 \mu \mathrm{L}\right.$ per well) and allowed to solidify $\left(37^{\circ}, 1 \mathrm{~h}\right)$ [27]. Then HMEC-1 cells or HUVEC cells $\left(2.5 \times 10^{4}\right.$ cells per well) were seeded onto the gel and cultured in the medium containing tanshinone- 1 at $37^{\circ}$ for $4 \mathrm{~h}$. The enclosed networks of complete tubes from three randomly chosen fields were counted and photographed under a microscope (Olympus, Tokyo, Japan).

\section{Cell migration assays}

The migration assay was done in a transwell Boyden chamber (Costar, MA, USA). HMEC-1 cells $\left(2 \times 10^{5}\right.$ cells per well $)$ in the serum-free MCDB131 medium containing tanshinone- $1(0,12.5,25$ or $50 \mu \mathrm{M})$ were added to the upper compartment of the chamber. The lower compartment contained MCDB131 medium supplemented with $20 \%$ fetal bovine serum (FBS) and 
tanshinone-1. After 6 -h incubation at $37^{\circ}$, the cells still staying on the upper face of the transwell membrane were removed with a cotton swab. The migrated cells on the lower side of the transwell membrane were fixed with $90 \%$ ethanol and then stained with $0.1 \%$ crystal violet in $0.1 \mathrm{M}$ borate and $2 \%$ ethanol $(\mathrm{pH} 9.0)$. After being photographed, the stained cells were subsequently extracted with $10 \%$ acetic acid. The absorbance values were determined at $600 \mathrm{~nm}$ with the SpectraMAX190 of Molecular Devices (Sunnyvale, CA).

\section{Aortic ring sprouting assays}

Aortic rings from 6-week-old Sprague Dawley rats $[28,29]$ were dissected into $1-\mathrm{mm}$-long rings. The rings were embedded with $70-\mu \mathrm{L}$ Matrigel, allowed to solidify $\left(37^{\circ}, 1 \mathrm{~h}\right)$ and then incubated with tanshinone- 1 in the serum-free M199 medium for 7 days. The aortic ring sprouts were photographed with a microscope (Olympus, Tokyo, Japan). All animal procedures and experiments complied with the ethical Guidelines for the care and use of animals and were approved by the Institute Animal Review Boards of the Shanghai Institute of Materia Medica at the Chinese Academy of Sciences.

\section{Chick chorioallantoic membrane assays}

Fertilized chicken eggs were incubated in an egg incubator (Lyon, CA) at $37^{\circ}$ and $50 \%$ humidity for 7 days. Then the alive embryos were chosen and the chorioallantoic membranes were treated with different concentrations of tanshinone-1 $(0,0.01,0.1,1,10$, $100 \mu \mathrm{M})$ dried on coverslips for $48 \mathrm{~h}$, as described previously [30]. The vascular images were captured by stereomicroscopic photography (MSS, Leica, Swizerland). Antiangiogenic effect was detected by counting the number of blood vessel branch points under the coverslips with tanshinone-1 (the right side of each image) or just with the vehicle (the left side of each image). At least 10 viable embryos were enrolled for each concentration group.

\section{Western blotting analyses}

The tested endothelial cells and tumor cells $\left(1.5 \times 10^{5}\right.$ cells per well) were exposed to different concentrations of tanshinone-1 at hypoxic or normoxic conditions for the indicated time. Then the cellular levels of proteins were determined as previously described [31-34].

\section{Real-time PCR}

Total RNA of cells was extracted using the TRIzol (Invitrogen, Carlsbad, CA, USA). One microgram of total RNA was reverse-transcribed using a RT reagent kit (TaKaRa, Tokyo, Japan).
The cDNA was amplified with a 7500 Fast Real-time PCR System (Applied Biosystem, Grand Island, NY, USA). The primer sequences were as follows: 5'-GCTGTCTTGGGTGCATTGGA-3' (forward) and 5'-ATGATTCTGCCCTCCTCCTTCT-3' (reverse) for vegf; 5'-CCAGCAGACTCA AATACAAGAACC-3' (forward) and 5'-TGTATGTGGGTAGGAGATGGAGAT-3' (reverse) for hif- $1 \alpha$; 5'-GGATGCAGAAGGAGATCACTG-3' (forward); and 5'-CGA TCCACACGGAGTACTTG-3' (reverse) for $\beta$-actin. The relative quantification of mRNA levels was automatically done with the software of the PCR system.

\section{ELISA kinase assays}

MCF-7 cells were seeded in a 6-well plate at a density of $1.5 \times 10^{5}$ cells per well. After attachment, the medium was replaced with fresh medium supplemented with $1 \%$ FBS, and the cells were treated with tanshinone-1 at hypoxia or normoxia for $16 \mathrm{~h}$. Cell supernatants were collected, and pellets were harvested by trypsinization to calculate the cell number. The amount of VEGF in the supernatant was determined with a human VEGF-ELISA kit (Multi Sciences, Shanghai, China) according to the manufacturer's instructions. VEGF was expressed as pictograms of VEGF protein per milliliter of medium and per $10^{5}$ cells.

\section{Small interfering RNA (siRNA)}

Cells were transfected with $100 \mathrm{nM}$ siRNA using RNAi Max (Invitrogen, Carlsbad, CA, USA) according to the manufacturer's instructions. The sequences of siRNA were as follows: 5'-GUUUGGAAAUAAU GGUGAATT-3' (siStat3-1) and 5'-GAGUUGAAUUAUCAGCUUATT-3' (siStat3-2) for stat3; 5'-CACCAUGAUAUGUUUACUATT-3' (siHIF$1 \alpha-1)$ and 5'-AGGACAAGUCACAACAGGAUU-3' (siHIF-1 $\alpha$-2) for hif-1 $\alpha$; and 5'-UUCUCCGAACGUGUC ACGUTT-3' as negative control (siCtrl). These siRNA sequences were synthesized by Shanghai GenePharma Co. Ltd (Shanghai, China).

\section{CONFLICTS OF INTEREST}

The authors declare no conflicts of interest related to this work.

\section{GRANT SUPPORT}

This work was supported by the Science and Technology Commission of Shanghai Municipality (No. 13XD1404200 to Z.H. Miao), the National Basic Research Program of China (No. 2012CB932502 to Z.H. Miao), the National Science \& Technology Major Project of China (No. 2012ZX09301-001-002 to Z.H. Miao), the National 
Natural Science Foundation of China (No. 81321092 to J. Ding), the "Interdisciplinary Cooperation Team" Program for Science and Technology Innovation of the Chinese Academy of Sciences to Z.H. Miao and the State Key Laboratory of Drug Research to Z.H. Miao.

\section{REFERENCES}

1. Jain RK. Antiangiogenesis strategies revisited: from starving tumors to alleviating hypoxia. Cancer Cell. 2014; 26:605-622.

2. Mullard A. Phase III setback for lead angiopoietin inhibitor. Nat Rev Drug Discov. 2014; 13:877-877.

3. Monk BJ, Poveda A, Vergote I, Raspagliesi F, Fujiwara K, BaeDS, Oaknin A, Ray-Coquard I, ProvencherDM, Karlan BY, Lhomme C, Richardson G, Rincon DG, et al. Antiangiopoietin therapy with trebananib for recurrent ovarian cancer (TRINOVA-1): a randomised, multicentre, doubleblind, placebo-controlled phase 3 trial. Lancet Oncol. 2014; 15:799-808.

4. Miao ZH, Feng JM, Ding J. Newly discovered angiogenesis inhibitors and their mechanisms of action. Acta Pharmacol Sin. 2012; 33:1103-1111.

5. Wang YQ, Miao ZH. Marine-derived angiogenesis inhibitors for cancer therapy. Mar Drugs. 2013; 11:903-933.

6. Yu B, Li MH, Wang W, Wang YQ, Jiang Y, Yang SP, Yue JM, Ding J, Miao ZH. Pseudolaric acid B-driven phosphorylation of c-Jun impairs its role in stabilizing HIF-1alpha: a novel function-converter model. J Mol Med (Berl). 2012; 90:971-981.

7. Yu B, Miao ZH, Jiang Y, Li MH, Yang N, Li T, Ding J. c-Jun protects hypoxia-inducible factor-1alpha from degradation via its oxygen-dependent degradation domain in a nontranscriptional manner. Cancer Res. 2009; 69:7704-7712.

8. Zhou ZL, Luo ZG, Yu B, Jiang Y, Chen Y, Feng JM, Dai M, Tong LJ, Li Z, Li YC, Ding J, Miao ZH. Increased accumulation of hypoxia-inducible factor-1alpha with reduced transcriptional activity mediates the antitumor effect of triptolide. Mol Cancer. 2010; 9:268.

9. Zhou ZL, Yang YX, Ding J, Li YC, Miao ZH. Triptolide: structural modifications, structure-activity relationships, bioactivities, clinical development and mechanisms. Nat Prod Rep. 2012; 29:457-475.

10. Dai M, Miao ZH, Ren X, Tong LJ, Yang N, Li T, Lin LP, Shen YM, Ding J. MFTZ-1 reduces constitutive and inducible HIF-1alpha accumulation and VEGF secretion independent of its topoisomerase II inhibition. J Cell Mol Med. 2010; 14:2281-2291.

11. Xu L, Feng JM, Li JX, Zhu JM, Song SS, Tong LJ, Chen Y, Yang XY, Shen YY, Lian FL, Li YP, Lin DH, Ding J, et al. Tanshinone-1 induces tumor cell killing, enhanced by inhibition of secondary activation of signaling networks. Cell Death Dis. 2013; 4:e905.
12. Dong Y, Morris-Natschke SL, Lee KH. Biosynthesis, total syntheses, and antitumor activity of tanshinones and their analogs as potential therapeutic agents. Nat Prod Rep. 2011; 28:529-542.

13. Wu WY, Wang YP. Pharmacological actions and therapeutic applications of Salvia miltiorrhiza depside salt and its active components. Acta Pharmacol Sin. 2012; 33:1119-1130.

14. Chen YJ, Chung TY, Chen WY, Chen CY, Lee MR, Jinn TR, Tc Tzen J. Detecting metabolites of different transition metal-lithospermate B complexes after intravenous injection in rats. Acta Pharmacol Sin. 2014; 35:937-944.

15. Nizamutdinova IT, Lee GW, Lee JS, Cho MK, Son KH, Jeon SJ, Kang SS, Kim YS, Lee JH, Seo HG, Chang KC, Kim HJ. Tanshinone I suppresses growth and invasion of human breast cancer cells, MDA-MB-231, through regulation of adhesion molecules. Carcinogenesis. 2008; 29:1885-1892.

16. Wang GL, Semenza GL. Purification and characterization of hypoxia-inducible factor 1. J Biol Chem. 1995; 270:1230-1237.

17. Yahata Y, Shirakata Y, Tokumaru S, Yamasaki K, Sayama K, Hanakawa Y, Detmar M, Hashimoto K. Nuclear translocation of phosphorylated STAT3 is essential for vascular endothelial growth factor-induced human dermal microvascular endothelial cell migration and tube formation. J Biol Chem. 2003; 278:40026-40031.

18. Carmeliet P, Ferreira V, Breier G, Pollefeyt S, Kieckens L, Gertsenstein M, Fahrig M, Vandenhoeck A, Harpal K, Eberhardt C, Declercq C, Pawling J, Moons L, et al. Abnormal blood vessel development and lethality in embryos lacking a single VEGF allele. Nature. 1996; 380:435-439.

19. Huang LE, Gu J, Schau M, Bunn HF. Regulation of hypoxia-inducible factor 1alpha is mediated by an O2-dependent degradation domain via the ubiquitinproteasome pathway. Proc Natl Acad Sci U S A. 1998; 95:7987-7992.

20. Yu H, Lee H, Herrmann A, Buettner R, Jove R. Revisiting STAT3 signalling in cancer: new and unexpected biological functions. Nat Rev Cancer. 2014; 14:736-746.

21. Atsumi T, Singh R, Sabharwal L, Bando H, Meng J, Arima Y, Yamada M, Harada M, Jiang JJ, Kamimura D, Ogura H, Hirano T, Murakami M. Inflammation amplifier, a new paradigm in cancer biology. Cancer Res. 2014; 74:8-14.

22. Middleton K, Jones J, Lwin Z, Coward JI. Interleukin-6: an angiogenic target in solid tumours. Crit Rev Oncol Hematol. 2014; 89:129-139.

23. Snyder M, Huang J, Huang XY, Zhang JJ. A signal transducer and activator of transcription 3. Nuclear Factor kappaB (Stat3.NFkappaB) complex is necessary for the expression of fascin in metastatic breast cancer cells in response to interleukin (IL)-6 and tumor necrosis factor (TNF)-alpha. J Biol Chem. 2014; 289:30082-30089. 
24. Xu Q, Briggs J, Park S, Niu G, Kortylewski M, Zhang S, Gritsko T, Turkson J, Kay H, Semenza GL, Cheng JQ, Jove R, $\mathrm{Yu}$ H. Targeting Stat3 blocks both HIF-1 and VEGF expression induced by multiple oncogenic growth signaling pathways. Oncogene. 2005; 24:5552-5560.

25. Miklossy G, Hilliard TS, Turkson J. Therapeutic modulators of STAT signalling for human diseases. Nat Rev Drug Discov. 2013; 12:611-629.

26. Li JX, Feng JM, Wang Y, Li XH, Chen XX, Su Y, Shen YY, Chen Y, Xiong B, Yang CH, Ding J, Miao ZH. The B-Raf(V600E) inhibitor dabrafenib selectively inhibits RIP3 and alleviates acetaminophen-induced liver injury. Cell Death Dis. 2014; 5:e1278.

27. Ren X, Dai M, Lin LP, Li PK, Ding J. Anti-angiogenic and vascular disrupting effects of $\mathrm{C} 9$, a new microtubuledepolymerizing agent. Br J Pharmacol. 2009; 156:1228-1238.

28. Nicosia RF, Ottinetti A. Growth of microvessels in serumfree matrix culture of rat aorta. A quantitative assay of angiogenesis in vitro. Lab Invest. 1990; 63:115-122.

29. Belleri M, Ribatti D, Nicoli S, Cotelli F, Forti L, Vannini V, Stivala LA, Presta M. Antiangiogenic and vasculartargeting activity of the microtubule-destabilizing transresveratrol derivative $3,5,4$ '-trimethoxystilbene. Mol Pharmacol. 2005; 67:1451-1459.
30. Ribatti D, Vacca A, Roncali L, Dammacco F. The chick embryo chorioallantoic membrane as a model for in vivo research on anti-angiogenesis. Curr Pharm Biotechnol. $2000 ; 1: 73-82$.

31. Miao ZH, Ding J. Transcription factor c-Jun activation represses mdr-1 gene expression. Cancer Res. 2003; 63:4527-4532.

32. Jiang Y, Miao ZH, Xu L, Yu B, Gong JX, Tong LJ, Chen Y, Zhou ZL, Liu HC, Wang Y, Guo YW, Ding J. Drug transporter-independent liver cancer cell killing by a marine steroid methyl spongoate via apoptosis induction. J Biol Chem. 2011; 286:26461-26469.

33. Manzo SG, Zhou ZL, Wang YQ, Marinello J, He JX, Li YC, Ding J, Capranico G, Miao ZH. Natural product triptolide mediates cancer cell death by triggering CDK7dependent degradation of RNA polymerase II. Cancer Res. 2012; 72:5363-5373.

34. Yi JM, Zhang XF, Huan XJ, Song SS, Wang W, Tian QT, Sun YM, Chen Y, Ding J, Wang YQ, Yang CH, Miao ZH. Dual Targeting of Microtubule and Topoisomerase II by $\alpha$-Carboline Derivative YCH337 for Tumor Proliferation and Growth Inhibition. Oncotarget. 2015 (in press). 